\title{
Precursors to invasive pancreatic cancer
}

This article was published in the following Dove Press journal:

Gastrointestinal Cancer:Targets and Therapy

24 August 2012

Number of times this article has been viewed

\section{Jessica A Smith \\ Aatur D Singhi \\ Anirban Maitra}

Department of Pathology, The Sol Goldman Pancreatic Cancer Research Center, Johns Hopkins University School of Medicine, Baltimore, MD, USA
Correspondence: Anirban Maitra The Johns Hopkins University School of Medicine, Department of Pathology, CRBII Room 34I 1550 Orleans Street, Baltimore, MD 21231, USA

Tel + I 4 I0 95535 I I

Email amaitral@jhmi.edu
Abstract: Pancreatic ductal adenocarcinoma (PDAC) is a lethal disease, with a 5-year survival rate of less than 5\%. To better understand PDAC and to improve its dismal prognosis, we must understand its origins. PDAC has three distinct noninvasive precursor lesions including intraductal papillary mucinous neoplasm, mucinous cystic neoplasm, and pancreatic intraepithelial neoplasia. Each of these precursor lesions has its own unique compendium of clinical findings, morphological features, and genetic aberrations. This review focuses on the clinical significance of precursor lesions of pancreatic cancer and how better understanding of these lesions can aid in early detection and treatment.

Keywords: pancreatic ductal adenocarcinoma, precursor lesions, intraductal papillary mucinous neoplasm, mucinous cystic neoplasm, pancreatic intraepithelial neoplasia, PanIN

\section{Introduction}

Pancreatic ductal adenocarcinoma (PDAC) is a nearly uniformly lethal cancer that remains the 4th leading cause of cancer death in the United States, with a 5-year survival rate of less than $5 \% .^{1}$ The dismal prognosis associated with PDAC is most likely attributed to the late clinical presentation, at which time the disease is usually at an advanced, and surgically inoperable, stage. ${ }^{2}$ Only a minority $(\sim 15 \%-20 \%)$ of patients with PDAC present with resectable disease, and the overwhelming majority are candidates for chemoradiation therapy only. These therapies can prolong survival, but rarely lead to a cure. Thus, one of the best opportunities for increasing the survival in PDAC is to detect the disease at an early and potentially noninvasive stage, the stage of precursor lesions. ${ }^{3,4}$ Enhancing our knowledge of the morphology, biological behavior, and molecular aberrations of these precursor lesions gives us more insight into the pathogenesis and natural history of this fatal disease.

PDAC is associated with multiple precancerous lesions: pancreatic intraepithelial neoplasia (PanIN), intraductal papillary mucinous neoplasm (IPMN), and mucinous cystic neoplasm (MCN). These are noninvasive epithelial neoplasms with an increased risk of transformation into PDAC. Each of these three entities exhibits a unique multistep morphologic and genetic progression to invasive adenocarcinoma. Herein, we review the clinicopathologic features, molecular aberrations, natural history, recommended treatment, and prognosis of these precursor lesions. 


\section{PanIN}

\section{Clinical features}

PanIN is the most common precursor lesion of PDAC. The term PanIN was adopted in 1999 by a Pancreas Cancer Think Tank sponsored by the National Cancer Institute to classify a spectrum of noninvasive intraductal lesions of the pancreas. ${ }^{5}$ PanINs are microscopic clonal lesions, located in the smaller pancreatic ducts, and measure less than $5 \mathrm{~mm}$ in diameter. ${ }^{5,6}$ The incidence of PanIN tends to increase with age, and both men and women are affected equally. ${ }^{7}$ PanINs are usually asymptomatic and isolated PanIN lesions are not detectable upon noninvasive abdominal imaging with rare exceptions. ${ }^{8}$ Andea et al found that PanINs were identified in $82 \%$ of pancreata with invasive carcinoma, in $60 \%$ of pancreata with chronic pancreatitis, and in $16 \%$ of otherwise normal pancreata resected secondary to trauma or benign pancreatic neoplasms. ${ }^{9}$ Of note, higher grade PanINs are almost exclusively found in association with invasive PDAC.

\section{Pathologic features}

Microscopically, PanIN lesions are characterized by cuboidal-to-columnar cells with varying amounts of cytoplasmic mucin and degrees of cytologic and architectural atypia (Figure 1A-D). The degree of dysplasia is used as a universal grading tool, and represents the progression from

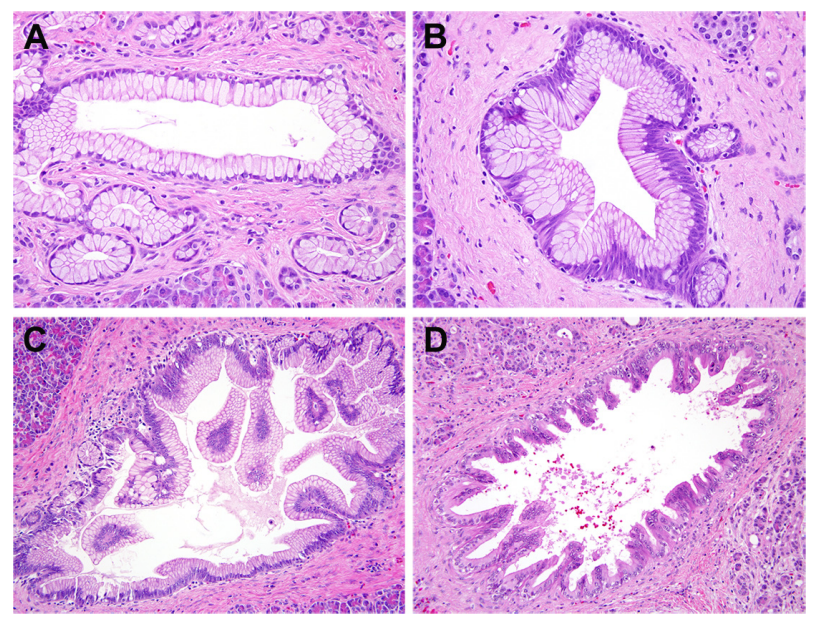

Figure I PanIN. (A) PanIN-IA is composed of flat columnar epithelium with abundant mucin and basally oriented, small, uniform nuclei. (B) PanIN-IB is identical to PanIN-IA, except it has either papillary or micropapillary architecture. (C) PanIN-2 often demonstrates papillary architecture and, by definition, has nuclear abnormalities that include moderate nuclear atypia, pseudostratification, loss of polarity, and hyperchromasia. Mitoses may be present, although rare, and are basally located and morphologically normal. (D) PanIN-3 is characterized by papillary or micropapillary architecture and marked nuclear atypia that is indistinguishable from invasive pancreatic ductal adenocarcinoma.

Note: However, it is confined to the basement membrane. Abbreviation: PanIN, pancreatic intraepithelial neoplasia. low-grade lesions to carcinoma in-situ/high-grade lesions. PanIN-1 lesions show minimal nuclear atypia, inconspicuous nucleoli, and absent mitotic figures (Figure $1 \mathrm{~A}$ and $\mathrm{B}$ ). PanIN-1 lesions are further classified based on architectural subtypes, flat (PanIN-1A) and papillary or micropapillary (PanIN-1B). Lesions with moderate nuclear atypia, pseudostratification, loss of polarity, and hyperchromasia are classified as PanIN-2 lesions (Figure 1C). Mitotic figures are rarely identified in PanIN-2 lesions. Marked atypia is characteristic of PanIN-3 lesions (Figure 1D). PanIN-3 lesions have a papillary, micropapillary, or occasional flat architecture. Cribriform structures may be present as well as necrosis and "budding" epithelial cells in the gland lumen. Unlike PanIN-1 and PanIN-2, mitotic figures, including atypical mitotic figures, are present. Yet PanIN-3 is confined to the basement membrane and no invasive growth is present. Immunohistochemical stains for PanINs show positive staining for MUC1, MUC5AC, and MUC6, particularly in the higher grade lesions. ${ }^{10-13}$ They are, however, negative for MUC2 and CDX2. The increasing grades of dysplasia in the PanIN lesions manifest the morphologic steps of tumor progression to invasive pancreatic cancer.

\section{Molecular features}

Molecular analysis of PanINs show similar genetic alterations to PDAC and can be divided into early and late events. Telomeric shortening is one of the earliest molecular aberrations detected in low-grade PanIN that likely facilitates genetic instability. ${ }^{14}$ Another early event is activating point mutations in the KRAS2 oncogene. Ultra-sensitive sequencing technologies applied to microdissected PanIN lesions have found near ubiquitous frequency of KRAS2 mutations in all histological grades, although the lower grade PanINs tend to be admixtures of cells with wild-type and mutant alleles. ${ }^{15}$ Later genetic events include inactivating mutations in $\mathrm{p16/}$ $C D K N 2 A$. Loss of 16 expression by immunohistochemistry can be found in $30 \%, 50 \%$, and $70 \%$ of PanIN-1, PanIN-2, and PanIN-3 lesions, respectively. ${ }^{16}$ Mutations in the tumor suppressor genes TP53, DPC4/SMAD4, and BRCA2 are late events and generally observed in PanIN-3 lesions. ${ }^{10}$ Loss of heterozygosity $(\mathrm{LOH})$ is observed, particularly in highergrade PanIN lesions, and at chromosomal loci harboring genes frequently mutated in PDAC. ${ }^{17}$ Of note, while most of the $\mathrm{LOH}$ events are concordant between the noninvasive precursor and adjacent PDAC, a minority of events can be discordant, reiterating that PanIN lesions arise as independent clones. In addition to gnomic aberrations, PanINs also accrue epigenetic abnormalities such as promoter hypermethylation 
and aberrant micro-RNA (miRNA) expression during the multistep progression to invasive PDAC. ${ }^{18,19}$

\section{Diagnosis, natural history, and treatment}

Due to their microscopic nature, PanINs are typically found incidentally in resection or in biopsy specimens, and are not associated with clinical signs or symptoms. Most PanINs are undetectable with modern imaging modalities, making early detection challenging. However, a recent study done by Brune et al and Detlefsen et al, showed that multifocal PanIN lesions, such as those arising in patients with familial pancreatic cancer, can be associated with parenchymal atrophy, which can be detected using endoscopic ultrasound (EUS). ${ }^{20,21}$ Anecdotal evidence suggests that higher-grade PanIN lesions that are left in situ upon partial pancreatectomy for PDAC can progress to adenocarcinoma within a timespan of months to a few years, underscoring the precursor nature of such lesions. ${ }^{22}$ Nonetheless, a recent study has demonstrated that the presence of PanIN lesions at the surgical margin of pancreata with otherwise invasive PDAC does not influence prognosis for the patient, and additional surgery is not required. ${ }^{23}$

\section{IPMN}

\section{Clinical features}

IPMNs are mucin-producing epithelial neoplasms that are characterized by intraductal proliferation of cells with varying degrees of atypia, which usually form papillae and lead to cystic dilation of the pancreatic ducts. ${ }^{24}$ By definition, IPMNs involve either the main pancreatic duct, or one of its branches, or both. These lesions arise within ducts that are greater than $1 \mathrm{~cm}$ in diameter, most frequently are located in the head of the pancreas, can be multifocal, and lack the ovarian-type stroma that is seen in MCNs. ${ }^{25} \mathrm{An}$ IPMN that is less than $1 \mathrm{~cm}$ in size is called an "incipient" IPMN ${ }^{26}$ These lesions are histologically identical to classical IPMNs, but fail to meet the size criteria. Men are more affected than women (3:2 ratio), and IPMNs tend to affect elderly patients with a mean age of diagnosis at 65 years (ranging in age from 25 to 95 years). Due to obstruction of the pancreatic ducts by mucin or the tumor itself, patients may present with intermittent episodes of pancreatitis, abdominal pain, nausea, and vomiting; other symptoms include weight loss, jaundice, and back pain.

\section{Pathologic features}

Noninvasive IPMNs are subdivided grossly into two groups based on the predominant site of involvement: main duct type (Figure 2A) and branch duct type (Figure 2B); ${ }^{27}$ although examples of mixed main and branch ducts also exist. Main duct IPMNs tend to have higher degrees of dysplasia, and are more often associated with invasive carcinoma ( $40 \%$ to $50 \%$ of cases). ${ }^{28,29}$ Microscopically, the epithelium lining the ducts is typically characterized by tall papillary structures with fibrovascular cores lined by a layer of mucin-secreting columnar epithelium with varying degrees of atypia. However, some subtypes, more often in branch duct IPMNs, may have flat epithelium. High-grade IPMNs show both increased cytologic atypia (nuclear pseudostratification and loss of polarity) and complex architectural patterns (branching papillae, pseudopapillary structures, and cribriform glands with neoplastic cells filling the lumen). High-grade IPMNs with complex papillary excrescences may fill the duct lumen leading to duct obstruction and proximal duct dilatation. Noninvasive IPMNs are graded on a histological spectrum, based on the degree of architectural and cytologic atypia, as low- (aka IPMN adenoma), intermediate-, and high-grade dysplasia (carcinoma in situ), respectively. ${ }^{30}$

IPMNs are generally classified into four different histologic subtypes based on their cytomorphological features: gastric foveolar type, intestinal type, pancreatobiliary type, and oncocytic type. ${ }^{24,31}$ Immunohistomchemical staining for mucin antibodies enables differentiation between the four types of IPMNs. Intestinal-type IPMNs are the most common and resemble villous adenomas found in the large intestine (Figure 3A). They form long finger-like projections that are lined by columnar mucin-rich neoplastic cells. By immunohistochemistry, intestinal-type IPMNs stain positive for both MUC2 and CDX2. The gastric-type IPMNs have an eosinophilc cytoplasm with abundant mucin and basally oriented nuclei (Figure 3B). Branch duct-type IPMNs often show gastric differentiation. Gastric-type IPMNs stain negative for both MUC1 and MUC2. The pancreatobiliary-type IPMNs
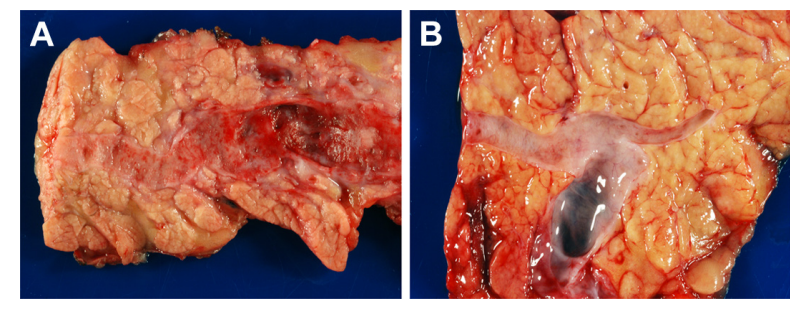

Figure 2 IPMN gross features. Grossly, IPMNs are classified based on their level of pancreatic duct involvement. (A) Those involving the main pancreatic duct are designated as main duct type and characterized by a dilated, tortuous main pancreatic duct. (B) IPMNs involving the secondary branches of the main pancreatic duct are designated as branch duct type.

Abbreviation: IPMN, intraductal papillary mucinous neoplasm. 


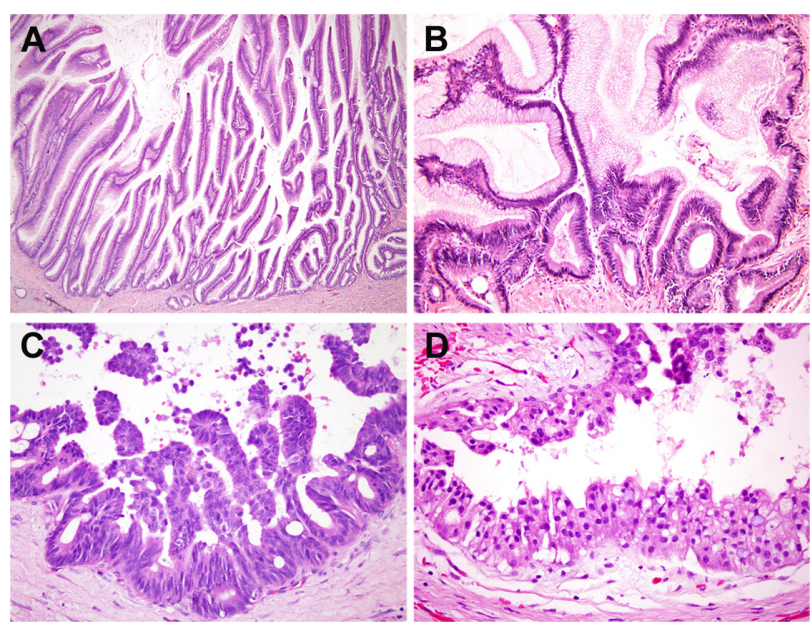

Figure 3 IPMN microscopic features. Microscopically, IPMNs are subdivided into four histologic subtypes. (A) Most IPMNs are of the intestinal type and characterized by long, villous finger-like projections, lined by columnar mucin-producing cells with cigar-shaped nuclei, resembling villous adenomas of the large intestine. (B) Gastric foveolar-type IPMNs demonstrate gastric foveolar differentiation with abundant apical cytoplasmic mucin and basally oriented nuclei. (C) The pancreatobiliary-type IPMN features complex papillae with bridging and cribriform architecture. The neoplastic cells are cuboidal with less cytoplasmic mucin than the intestinal- and gastric-type IPMNs and hyperchromatic nuclei. (D) Oncocytic-type IPMNs are architecturally complex with arborizing papillae and cribriform architecture.

Note: As the name implies, oncocytic-type IPMNs are lined by cuboidal cells with prominent oncocytic granular cytoplasm and enlarged, hyperchromatic nuclei.

Abbreviation: IPMN, intraductal papillary mucinous neoplasm.

often demonstrate moderate-to-marked nuclear atypia and more complex architecture with papillae forming bridges and a cribriform pattern (Figure 3C). Immunohistochemically, the pancreatobiliary-type stains positive for MUC1. The last subtype of IPMNs is the oncocytic subtype, which contains abundant mitochondria imparting a distinctive eosinophilic appearance to the cytoplasm (Figure 3D). The nuclei are large and have prominent nucleoli, and the cytoplasm often stains positive for MUC1. These subtypes of IPMNs have clinical and prognostic relevance, for example the gastric subtype is associated with the better prognosis, followed by oncocytic and intestinal subtypes, while the pancreatobiliary type is associated with a poor prognosis and adverse clinical behavior. ${ }^{31}$ It is important to note that IPMNs can consist of a mixture of subtypes.

One-third of IPMNs, usually main duct, are associated with invasive pancreatic adenocarcinoma. ${ }^{32}$ Three different histological subtypes of adenocarcinoma have been described: colloid, oncocytic, and ductal adenocarcinoma; with colloid being the most common of the three. Intestinaltype IPMNs often progress to colloid carcinoma, while pancreatobiliary-type IPMNs more often develop ductal adenocarcinoma. Invasive adenocarcinoma arising from oncocytic IPMNs are typically oncocytic rather than ductal or colloid. IPMNs can also be associated with extrapancreatic malignancies including gastric and colorectal carcinoma. ${ }^{33}$

\section{Molecular features}

Unlike PanINs, MCNs, and PDAC, DPC4/SMAD4 gene mutations are an uncommon finding in IPMNs. ${ }^{34} \mathrm{KRAS2}$ mutations increase with increasing degrees of dysplasia, and TP53 and $p 16 / C D K N 2 A$ gene inactivation, both commonly found in PDAC, can vary among IPMN cases. Interestingly loss of expression of the protein product of $S T K 11 / L K B 1$, a gene associated with Peutz-Jeghers syndrome, is observed in $25 \%$ of IPMNs, ${ }^{35}$ and the PIK $3 C A$ gene is mutated in $10 \%$ of cases. ${ }^{36}$ In a recent study, Wu et al identified recurrent point mutations of GNAS at codon 201 in $60 \%$ of IPMNs. ${ }^{37}$ GNAS encodes for the alpha subunit of a stimulatory G-protein, which in turn, activates cyclic AMP pathways within cells. ${ }^{38}$ Interestingly, GNAS mutations were more often seen in intestinal-type IPMNs. Furukawa et al evaluated the rate of mutations involving codon 201 of GNAS in 118 IPMNs and 32 conventional pancreatic adenocarcinoma cases, and found that $\sim 40 \%$ of the IPMNs, and none of the pancreatic adenocarcinomas, harbored GNAS mutations. ${ }^{39}$ The G-protein alpha unit and downstream effectors, including phosphorylated substrates of protein kinase A (PKA), were expressed in the IPMN tissues bearing mutant GNAS. The frequency of GNAS mutations did not vary with grades of IPMN dysplasia however the expression of PKA was associated with neoplastic grade. PKA expression was greater in high-grade and invasive IPMNs versus low-grade IPMNs. These findings suggest that $G N A S$ mutations are unique to IPMNs and play a role in their pathogenesis and that activation of G-protein alpha and downstream components may contribute to the progression of IPMNs.

\section{Diagnosis, natural history, and treatment}

A finding, that is strongly consistent with IPMNs, is mucin extruding from a patulous ampulla of Vater on upper endoscopy. Although atypical cells and mucin may be found on endoscopic ultrasound-guided fine-needle aspiration, the sensitivity of aspiration cytology in cystic lesions of the pancreas is suboptimal. ${ }^{40,41}$ Imaging modalities may be rather helpful in the diagnosis of IPMNs. ${ }^{42-44} \mathrm{CT}$ and endoscopic retrograde cholangiopancreatography usually reveal dilated main and branched pancreatic ducts, due to obstruction of the lumen by mucous plugs and papillary projections of the tumor. Plugging of the ductal lumina leads to the formation of grape-like cystic lesions that are diagnostic of IPMN lesions. Magnetic resonance cholangiopancreatography may also be 
a useful tool, and usually demonstrates ductal dilation and occasionally mural nodules. ${ }^{45}$

The natural history of noninvasive IPMNs and the precise timeline for their progression to invasive cancer is not well known, because the majority of large and symptomatic lesions are removed before invasion. Nonetheless, given that many patients report a long history of symptoms before being diagnosed with an invasive component associated with an IPMN suggests that the natural history is at least 5 to 10 years for such a progression. ${ }^{30,46}$ Features suggestive of malignant IPMNs include: main duct IPMN, clinical symptoms, cysts greater than $3 \mathrm{~cm}$, mural nodules, lymphadenopathy, distension of the pancreatic main duct, positive cytology on EUS, and a CEA level greater than 200 in the cystic contents. ${ }^{24,28}$

Main-duct IPMNs have a greater risk of malignancy, approaching $40 \%-50 \%$, while patients with branch duct IPMNs are at a much lower risk of malignant transformation with a risk $\sim 10 \%$ (the range is somewhat variable based on the specific series). ${ }^{29,47-50}$ Surgery remains the standard of care in the management of main duct IPMNs given their malignant potential. ${ }^{51}$ Treatment of IPMNs involving the branch ducts is somewhat controversial, in terms of definitive surgery versus conservative follow up and management. High risk features and indications for surgery include: size $>3 \mathrm{~cm}$, symptoms, and suspicious radiological findings such as solid or mural nodules and distension of the main pancreatic duct ("Sendai criteria"). ${ }^{28}$ However, preoperative radiological assessments in asymptomatic branch duct IPMNs are not precise in up to one-third of cases, underscoring the need for ancillary diagnostic parameters like mutational analysis in cyst fluid. ${ }^{52}$ It is important to note that surgical resection does not always result in a definite cure due to the fact that noninvasive IPMNs can be multifocal and recur. ${ }^{53} \mathrm{~A}$ small percentage of patients develop a second pancreatic neoplasm, which can be fatal. Therefore, careful follow up and clinical management is needed after resection of IPMNs to evaluate for metachronous disease.

\section{MCNs}

\section{Clinical features}

MCNs are mucin-producing, cyst-forming epithelial neoplasms, most commonly arising in the body/tail of the pancreas, and they are supported by a distinctive ovarian-type stroma. ${ }^{30}$ These lesions are almost exclusively diagnosed in young women with a mean age of 40 to 50 years (ranging in age from 14 to 95 years). MCNs can be found incidentally in asymptomatic patients on a physical exam or by imaging and may also be detected in symptomatic patients. ${ }^{54}$
Patients most often present with epigastric pain, a sense of abdominal fullness, nausea and vomiting, diarrhea, or symptoms due to a mass effect. Approximately 5\% to $30 \%$ of symptomatic patients might harbor a palpable mass upon physical examination. Patients with MCNs usually have normal CA 19-9 levels, unless the MCN is accompanied by an invasive adenocarcinoma.

\section{Pathologic features}

MCNs are predominately identified in the body/tail of the pancreas. MCNs can be substantially large in size, ranging in diameter from $1.5 \mathrm{~cm}$ to $30 \mathrm{~cm}$ in size. Grossly, less than $20 \%$ of MCNs communicate with the pancreatic duct. ${ }^{55}$ The cyst surface is smooth and glistening, and covered with large dilated blood vessels and a thick fibrous capsule (Figure 4A). MCNs tend to be multilocular, are rarely multifocal, and contain abundant mucin and/or hemorrhagic fluid (Figure 4B to D). Microscopically, the cysts are lined by columnar mucin-producing epithelium arranged in a flat architecture (Figure 5A and B). However, low papillary projections, cribriform, and glandular formation may be present, especially in high-grade neoplasms. As mentioned above, these lesions have a distinctive ovarian type stroma, which forms a "Grenz" zone underneath the epithelium, and can be highlighted with immunohistochemical stains for PR, ER, and inhibin A (Figure 5B).

These neoplasms, like the other precursor lesions, are graded based on cytologic and architectural atypia. Lowgrade MCNs are composed of a uniform single-layer of benign mucinous columnar cells; the nuclei are basally
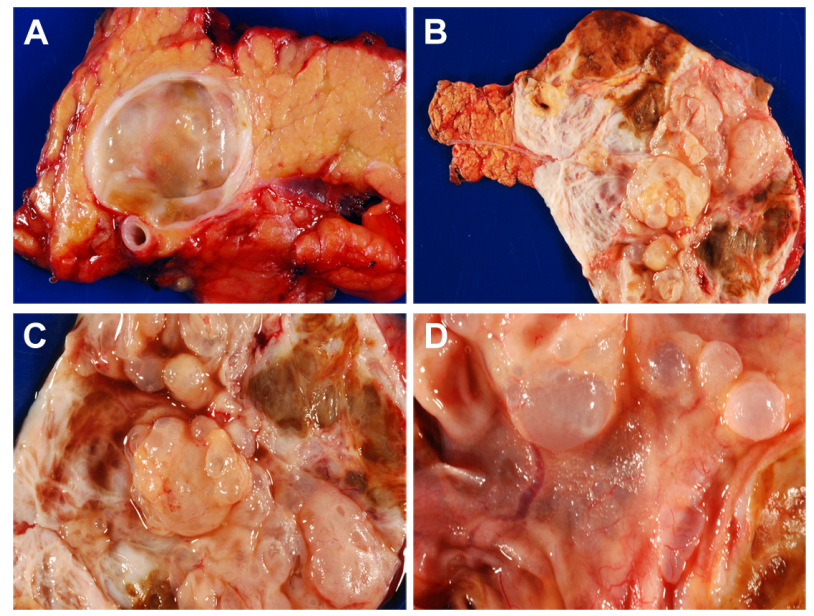

Figure $4 \mathrm{MCN}$ gross features. Grossly, MCNs are solitary lesions that do not communicate with the pancreatic ducts. They may be either (A) unilocular or (B) multilocular. (C and D) The locules have thick walls and are filled with thick tenacious mucin, although some may be filled with hemorrhagic fluid.

Abbreviation: MCN, mucinous cystic neoplasm. 


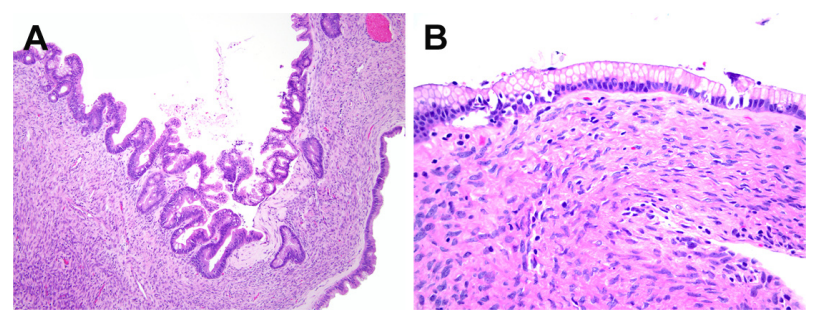

Figure 5 MCN microscopic features. (A and B) Microscopically, the cysts in MCNs are lined by tall columnar, mucin producing with basally oriented nuclei, and abundant intracytoplasmic mucin.

Notes: Underlying the mucinous epithelium is a densely cellular spindled stroma with sparse cytoplasm and wavy nuclei termed ovarian type stroma. The ovarian type stroma is a distinctive and even diagnostic finding in establishing the diagnosis of MCN.

Abbreviation: MCN, mucinous cystic neoplasm.

located, small, and uniform in size. Intermediate- and highgrade MCNs consist of columnar epithelium with varying degrees of nuclear atypia with loss of nuclear polarity. Typically, the tumor epithelium can have a spectrum of different degrees of dysplasia, and the tumor should be graded as the highest grade present, even if only focal.

\section{Molecular features}

Early molecular events in the pathogenesis of MCNs include activating point mutations in KRAS2 and late events include TP53 and DPC4/SMAD4 mutations. ${ }^{56}$ Also documented in a minority of MCNs is the aberrant methylation of $p 16 /$ $C D K N 2 A .{ }^{56}$ However, a recent study identified that MCNs often harbor mutations of RNF43 with accompanying $\mathrm{LOH}$ of the wild-type allele. ${ }^{57} R N F 43$ encodes for an E3 ubiquitin ligase, and recent data suggests that the encoded protein leads to accelerated turnover of the Wnt receptor and therefore antagonizes oncogenic Wnt signaling. ${ }^{58}$ Of note, overall, approximately $50 \%$ of pancreatic mucinous cysts - IPMNs and MCNs - harbor inactivate mutations of RNF43. It is unclear whether disruption of RNF43 activity leads to oncogenic Wnt signaling in exocrine cystic neoplasms, or other targets of this ubiquitin ligase are aberrantly activated.

\section{Diagnosis, natural history, and treatment}

Imaging studies are the best diagnostic tool for MCN lesions. ${ }^{55,59} \mathrm{CT}$ often reveals well-circumscribed thickwalled multilocular cysts, ranging in size from 1 to $3 \mathrm{~cm}$, typically located in the tail of the pancreas. Occasionally, noninvasive $\mathrm{MCNs}$ can be differentiated from invasive MCNs based on the finding of mural nodules within the cysts, which are more common in invasive lesions. Endoscopic retrograde cholangiopancreatography is another diagnostic method used to detect MCNs. The large cystic structures often displace or compress the pancreatic duct, and in the majority of cases the cysts do not communicate with the pancreatic ducts..$^{55}$

MCNs progress to invasive carcinoma less often than IPMNs. Approximately $6 \%$ to $36 \%$ of MCNs are associated with an invasive component at the time of resection. ${ }^{60}$ Due to their malignant potential, all MCNs should be removed to prevent further progression. The disease-specific 5-year survival approaches $100 \%$ for patients with a noninvasive resected MCN. If an invasive component is present, the 5-year survival decreases to approximately $50 \%$ to $60 \%{ }^{61}$ Of special note, invasive adenocarcinomas usually arise focally in MCNs and complete resection of the MCN lesion is recommended. Partial resections of MCNs should be avoided to prevent missing an invasive component. If detected early and completely excised, MCNs are potentially curable neoplasms.

\section{Familial pancreatic cancer}

Approximately $10 \%$ of patients with pancreatic carcinoma have a known family history. ${ }^{63}$ Individuals with a first-degree relative with pancreatic cancer are thought to have a twofold increase in risk of developing pancreatic cancer, and this risk increases with each first-degree relative with a pancreatic cancer history. ${ }^{63}$ Understanding the genetic defects and precursor lesions associated with familial pancreatic carcinoma could therefore help explain the pathogenesis of this disease and aid in better screening and prophylactic methods. In a recent study done by Shi et al, noninvasive precursor lesions were prevalent in the resected pancreata from patients with familial pancreatic carcinoma, but they were found more often than those with sporadic disease, and were of higher grade.$^{63}$ It is recommended that high-risk individuals with a family history of pancreatic cancer should be screened routinely for precursor lesions. ${ }^{64}$

\section{Future directions}

The vast majority of pancreatic cancer patients are diagnosed at an advanced stage or after metastatic dissemination. Detecting these tumors at the precursor level could help alleviate the poor prognosis associated with pancreatic cancer. Cystic pancreatic lesions (IPMNs and MCNs) can be detected using a wide variety of imaging techniques, either incidentally, or when they become symptomatic. PanIN lesions however, are, for the most part, too small to be identified using current imaging modalities. One of the challenges for the future is to be able to detect higher-grade PanIN lesions prior to acquisition of invasive properties.

A recent genetic study has demonstrated a large window of opportunity where pancreatic cancer can potentially be 
detected prior to becoming invasive and terminally metastatic. In fact, one of the best opportunities for curing pancreatic cancer remains its early detection, given that $80 \%$ or more of patients are currently diagnosed at a surgically unresectable stage. One potential molecular target for screening is mutant $K R A S 2$ DNA, which is shed by neoplastic cells into blood, pancreatic juice, or stool. ${ }^{65,66}$ The near ubiquitous nature of $K R A S 2$ mutations renders this as an attractive marker for pancreatic cancer detection in peripheral biospecimens. However, KRAS2 mutations per se are not specific to invasive pancreatic cancer, and may occur in patients with low-grade precursor lesions as well as those with chronic pancreatitis; $;{ }^{67}$ thus, any tractable assay cannot be a simple "yes/no" readout but must also quantify the amount of mutant DNA within the biospecimen of interest. ${ }^{65,66}$ Other common somatic mutational events, such as TP53 and CDKN2A/p16 mutations are also amenable to detection in biospecimens like pancreatic juice, as are epigenetic alterations such as aberrant promoter methylation. ${ }^{68,69}$ For pancreatic cystic lesions, EUS and aspiration of cyst fluid contents provides an opportunity for molecular analysis, such as quantitative assessment of mutant DNA using next generation sequencing, ${ }^{37}$ or multiplex microRNA profiling for aberrant biomarker expression. ${ }^{70}$ Ultimately, there is unlikely to be one biomarker that proves to be the panacea for early detection, and most likely, we will utilize a multianalyte panel (encompassing DNA, RNA, proteins, or any combinations thereof) for the early diagnosis and therapeutic stratification of pancreatic neoplasia.

\section{Conclusion}

Our understanding and knowledge of precursor lesions of pancreatic cancer and their morphological appearance, biological behavior, molecular abnormalities, and clinical implications has significantly progressed over the years. We are now poised to apply the lessons learned over the past decade in precursor lesions of pancreatic cancer towards improving the diagnosis and survival of patients with this lethal malignancy. Ongoing advances in modern imaging techniques and molecular screening tools for high risk individuals provide an unprecedented opportunity for detecting and treating precursor lesions prior to their progression to invasive adenocarcinoma.

\section{Disclosure}

The authors report no conflicts of interest in this work.

\section{References}

1. Vincent A, Herman J, Schulick R, Hruban RH, Goggins M. Pancreatic cancer. Lancet. 2011;378(9791):607-620.
2. Yachida S, Jones S, Bozic I, et al. Distant metastasis occurs late during the genetic evolution of pancreatic cancer. Nature. 2010;467(7319): 1114-1117.

3. Singh M, Maitra A. Precursor lesions of pancreatic cancer: molecular pathology and clinical implications. Pancreatology. 2007;7(1): 9-19.

4. Takaori K. Current understanding of precursors to pancreatic cancer J Hepatobiliary Pancreat Surg. 2007;14(3):217-223.

5. Hruban RH, Adsay NV, Albores-Saavedra J, et al. Pancreatic intraepithelial neoplasia: a new nomenclature and classification system for pancreatic duct lesions. Am J Surg Pathol. 2001;25(5):579-586.

6. Hruban RH, Takaori K, Klimstra DS, et al. An illustrated consensus on the classification of pancreatic intraepithelial neoplasia and intraductal papillary mucinous neoplasms. Am J Surg Pathol. 2004;28(8): 977-987.

7. Cubilla AL, Fitzgerald PJ. Morphological lesions associated with human primary invasive nonendocrine pancreas cancer. Cancer Res. 1976;36(7 PT 2):2690-2698.

8. Takaori K, Matsusue S, Fujikawa T, et al. Carcinoma in situ of the pancreas associated with localized fibrosis: a clue to early detection of neoplastic lesions arising from pancreatic ducts. Pancreas. 1998;17(1): 102-105.

9. Andea A, Sarkar F, Adsay VN. Clinicopathological correlates of pancreatic intraepithelial neoplasia: a comparative analysis of 82 cases with and 152 cases without pancreatic ductal adenocarcinoma. Mod Pathol. 2003;16(10):996-1006.

10. Maitra A, Adsay NV, Argani P, et al. Multicomponent analysis of the pancreatic adenocarcinoma progression model using a pancreatic intraepithelial neoplasia tissue microarray. Mod Pathol. 2003;16(9): 902-912.

11. Adsay NV, Merati K, Andea A, et al. The dichotomy in the preinvasive neoplasia to invasive carcinoma sequence in the pancreas: differential expression of MUC1 and MUC2 supports the existence of two separate pathways of carcinogenesis. Mod Pathol. 2002;15(10):1087-1095.

12. Moriya T, Kimura W, Semba S, et al. Biological similarities and differences between pancreatic intraepithelial neoplasias and intraductal papillary mucinous neoplasms. Int J Gastrointest Cancer. 2005;35(2): 111-119.

13. Nagata K, Horinouchi M, Saitou M, et al. Mucin expression profile in pancreatic cancer and the precursor lesions. J Hepatobiliary Pancreat Surg. 2007;14(3):243-254.

14. van Heek NT, Meeker AK, Kern SE, et al. Telomere shortening is nearly universal in pancreatic intraepithelial neoplasia. Am J Pathol. 2002;161(5):1541-1547.

15. Kanda M, Matthaei H, Wu J, et al. Presence of somatic mutations in most early-stage pancreatic intraepithelial neoplasia. Gastroenterology. 2012;142(4):730-733.

16. Wilentz RE, Geradts J, Maynard R, et al. Inactivation of the p16 (INK4A) tumor-suppressor gene in pancreatic duct lesions: loss of intranuclear expression. Cancer Res. 1998;58(20):4740-4744.

17. Yamano M, Fujii H, Takagaki T, Kadowaki N, Watanabe H, Shirai T. Genetic progression and divergence in pancreatic carcinoma. Am J Pathol. 2000;156(6):2123-2133.

18. Ryu JK, Hong SM, Karikari CA, Hruban RH, Goggins MG, Maitra A. Aberrant MicroRNA-155 expression is an early event in the multistep progression of pancreatic adenocarcinoma. Pancreatology. 2010;10(1): 66-73.

19. Sato N, Fukushima N, Hruban RH, Goggins M. CpG island methylation profile of pancreatic intraepithelial neoplasia. Mod Pathol. 2008;21(3): 238-244.

20. Brune K, Abe T, Canto M, et al. Multifocal neoplastic precursor lesions associated with lobular atrophy of the pancreas in patients having a strong family history of pancreatic cancer. Am J Surg Pathol. 2006; 30(9):1067-1076.

21. Detlefsen S, Sipos B, Feyerabend B, Klöppel G. Pancreatic fibrosis associated with age and ductal papillary hyperplasia. Virchows Arch. 2995;447:800-805. 
22. Brat DJ, Lillemoe KD, Yeo CJ, Warfield PB, Hruban RH. Progression of pancreatic intraductal neoplasias to infiltrating adenocarcinoma of the pancreas. Am J Surg Pathol. 2005;447(5):800-805.

23. Matthaei H, Hong SM, Mayo SC, et al. Presence of pancreatic intraepithelial neoplasia in the pancreatic transection margin does not influence outcome in patients with R0 resected pancreatic cancer. Ann Surg Oncol. 2011;18(12):3493-3499.

24. Furukawa T, Klöppel G, Volkan Adsay N, et al. Classification of types of intraductal papillary-mucinous neoplasm of the pancreas: a consensus study. Virchows Arch. 2005;447(5):794-799.

25. Hruban RH, Takaori K, Klimstra DS, et al. An illustrated consensus on the classification of pancreatic intraepithelial neoplasia and intraductal papillary mucinous neoplasms. Am J Surg Pathol. 2004;28(8): 977-987.

26. Shi C, Klein AP, Goggins M, et al. Increased prevalence of precursor lesions in familial pancreatic cancer patients. Clin Cancer Res. 2009; 15(24):7737-7743.

27. Kobari M, Egawa S, Shibuya K, et al. Intraductal papillary mucinous tumors of the pancreas comprise 2 clinical subtypes: differences in clinical characteristics and surgical management. Arch Surg. 1999;134(10): $1131-1136$

28. Tanaka M, Chari S, Adsay V, et al; for International Association of Pancreatology. International consensus guidelines for management of intraductal papillary mucinous neoplasms and mucinous cystic neoplasms of the pancreas. Pancreatology. 2006;6(1-2):17-32.

29. Salvia R, Fernández-del Castillo C, Bassi C, et al. Main-duct intraductal papillary mucinous neoplasms of the pancreas: clinical predictors of malignancy and long-term survival following resection. Ann Surg. 2004; 239(5):678-685.

30. Hruban RH, Pitman MB, Klimstra DS. Tumors of the Pancreas. Washington, DC: American Registry of Pathology; 2006.

31. Ishida M, Egawa S, Aoki T, et al. Characteristic clinicopathological features of the types of intraductal papillary-mucinous neoplasms of the pancreas. Pancreas. 2007;35(4):348-352.

32. Fernández-del Castillo C, Adsay NV. Intraductal papillary mucinous neoplasms of the pancreas. Gastroenterology. 2010;139(3):708-713, 713. e1-e2.

33. Ishida M, Egawa S, Kawaguchi K, et al. Synchronous and metachronous extrapancreatic malignant neoplasms in patients with intraductal papillary-mucinous neoplasm of the pancreas. Pancreatology. 2008;8(6): $577-582$.

34. Iacobuzio-Donahue CA, Klimstra DS, Adsay NV, et al. Dpc-4 protein is expressed in virtually all human intraductal papillary mucinous neoplasms of the pancreas: comparison with conventional ductal adenocarcinomas. Am J Pathol. 2000;157(3):755-761.

35. Sahin F, Maitra A, Argani P, et al. Loss of Stk11/Lkb1 expression in pancreatic and biliary neoplasms. Mod Pathol. 2003;16(7): 686-691.

36. Schönleben F, Allendorf JD, Qiu W, et al. Mutational analyses of multiple oncogenic pathways in intraductal papillary mucinous neoplasms of the pancreas. Pancreas. 2008;36(2):168-172.

37. Wu J, Matthaei H, Maitra A, et al. Recurrent GNAS mutations define an unexpected pathway for pancreatic cyst development. Sci Transl Med. 2011;3(92):92ra66.

38. Weinstein LS, Liu J, Sakamoto A, Xie T, Chen M. Minireview: GNAS: normal and abnormal functions. Endocrinology. 2004;145(12): 5459-5464.

39. Furukawa T, Kuboki Y, Tanji E, et al. Whole-exome sequencing uncovers frequent GNAS mutations in intraductal papillary mucinous neoplasms of the pancreas. Sci Rep. 2011;1:161.

40. Pais SA, Attasaranya S, Leblanc JK, Sherman S, Schmidt CM, DeWitt J. Role of endoscopic ultrasound in the diagnosis of intraductal papillary mucinous neoplasms: correlation with surgical histopathology. Clin Gastroenterol Hepatol. 2007;5(4):489-495.

41. Vander Noot MR 3rd, Eloubeidi MA, Chen VK, et al. Diagnosis of gastrointestinal tract lesions by endoscopic ultrasound-guided fine-needle aspiration biopsy. Cancer. 2004;102(3):157-163.
42. Guarise A, Faccioli N, Ferrari M, et al. Evaluation of serial changes of pancreatic branch duct intraductal papillary mucinous neoplasms by follow-up with magnetic resonance imaging. Cancer Imaging. 2008;8: 220-228.

43. Bassi C, Sarr MG, Lillemoe KD, Reber HA. Natural history of intraductal papillary mucinous neoplasms (IPMN): current evidence and implications for management. J Gastrointest Surg. 2008;12(4):645-650.

44. Yamaguchi K, Tanaka M. Radiologic imagings of cystic neoplasms of the pancreas. Pancreatology. 2001;1(6):633-636.

45. Yoon LS, Catalano OA, Fritz S, Ferrone CR, Hahn PF, Sahani DV. Another dimension in magnetic resonance cholangiopancreatography: comparison of 2- and 3-dimensional magnetic resonance cholangiopancreatography for the evaluation of intraductal papillary mucinous neoplasm of the pancreas. J Comput Assist Tomogr. 2009;33(3): 363-368.

46. Crippa S, Fernández-Del Castillo C, Salvia R, et al. Mucin-producing neoplasms of the pancreas: an analysis of distinguishing clinical and epidemiologic characteristics. Clin Gastroenterol Hepatol. 2010;8(2): 213-219.

47. Allen PJ, Brennan MF. The management of cystic lesions of the pancreas. Adv Surg. 2007;41:211-228.

48. Sugiyama M, Suzuki Y, Abe N, Mori T, Atomi Y. Management of intraductal papillary mucinous neoplasm of the pancreas. J Gastroenterol. 2008;43(3):181-185.

49. Rodriguez JR, Salvia R, Crippa S, et al. Branch-duct intraductal papillary mucinous neoplasms: observations in 145 patients who underwent resection. Gastroenterology. 2007;133(1):72-79.

50. Salvia R, Crippa S, Falconi M, et al. Branch-duct intraductal papillary mucinous neoplasms of the pancreas: to operate or not to operate? Gut. 2007;56(8):1086-1090.

51. Schnelldorfer T, Sarr MG, Nagorney DM, et al. Experience with 208 resections for intraductal papillary mucinous neoplasm of the pancreas. Arch Surg. 2008;143(7):639-646.

52. Correa-Gallego C, Ferrone CR, Thayer SP, et al. Incidental pancreatic cysts: do we really know what we are watching? Pancreatology. 2010; 10(2-3):144-150.

53. Salvia R, Partelli S, Crippa S, et al. Intraductal papillary mucinous neoplasms of the pancreas with multifocal involvement of branch ducts. Am J Surg. 2009;198(5):709-714

54. Fernández-del Castillo C. Mucinous cystic neoplasms. J Gastrointest Surg. 2008;12(3):411-413.

55. Yamao K, Yanagisawa A, Takahashi K, et al. Clinicopathological features and prognosis of mucinous cystic neoplasm with ovariantype stroma: a multi-institutional study of the Japan pancreas society. Pancreas. 2011;40(1):67-71.

56. Scarlett CJ, Salisbury EL, Biankin AV, Kench J. Precursor lesions in pancreatic cancer: morphological and molecular pathology. Pathology. 2011;43(3):183-200.

57. Wu J, Jiao Y, Dal Molin M, et al. Whole-exome sequencing of neoplastic cysts of the pancreas reveals recurrent mutations in components of ubiquitin-dependent pathways. Proc Natl Acad Sci U SA. 2011;108(52): 21188-21193.

58. Hao HX, Xie Y, Zhang Y, et al. ZNRF3 promotes Wnt receptor turnover in an R-spondin-sensitive manner. Nature. 2012;485(7397):195-200.

59. Scott J, Martin I, Redhead D, Hammond P, Garden OJ. Mucinous cystic neoplasms of the pancreas: imaging features and diagnostic difficulties. Clin Radiol. 2000;55(3):187-192.

60. Wilentz RE, Albores-Saavedra J, Hruban RH. Mucinous cystic neoplasms of the pancreas. Semin Diagn Pathol. 2000;17(1):31-42.

61. Matthaei H, Schulick RD, Hruban RH, Maitra A. Cystic precursors to invasive pancreatic cancer. Nat Rev Gastroenterol Hepatol. 2011;8(3): $141-150$.

62. Shi C, Hruban RH, Klein AP. Familial pancreatic cancer. Arch Pathol Lab Med. 2009;133(3):365-374.

63. Shi C, Klein AP, Goggins M, et al. Increased prevalence of precursor lesions in familial pancreatic cancer patients. Clin Cancer Res. 2009; 15(24):7737-7743. 
64. Canto MI, Goggins M, Hruban RH, et al. Screening for early pancreatic neoplasia in high-risk individuals: a prospective controlled study. Clin Gastroenterol Hepatol. 2006;4(6):766-781.

65. Shi C, Fukushima N, Abe T, et al. Sensitive and quantitative detection of KRAS2 gene mutations in pancreatic duct juice differentiates patients with pancreatic cancer from chronic pancreatitis, potential for early detection. Cancer Biol Ther. 2008;7(3):353-360.

66. Shi C, Fukushima N, Abe T, et al. Sensitive and quantitative detection of KRAS2 gene mutations in pancreatic duct juice differentiates patients with pancreatic cancer from chronic pancreatitis, potential for early detection. Cancer Biol Ther. 2008;7(3):353-360.

67. Marchese R, Muleti A, Brozzetti S, Gandini O, Brunetti E, French D. Low value of detection of KRAS2 mutations in circulating DNA to differentiate chronic pancreatitis to pancreatic cancer. $\mathrm{Br} J$ Cancer. 2004;90(11):2243
68. Bian Y, Matsubayashi H, Li CP, et al. Detecting low-abundance p16 and p53 mutations in pancreatic juice using a novel assay: heteroduplex analysis of limiting dilution PCRs. Cancer Biol Ther. 2006;5(10): 1392-1399.

69. Fukushima N, Walter KM, Uek T, et al. Diagnosing pancreatic cancer using methylation specific PCR analysis of pancreatic juice. Cancer Biol Ther. 2003;2(1):78-83.

70. Matthaei H, HM, Wylie D, Lloyd MB, et al. MicroRNA biomarkers in cyst fluid augment the diagnosis and management of pancreatic cysts. Clin Cancer Res. June 21, 2012. [Epub ahead of print.]

\section{Publish your work in this journal}

Gastrointestinal Cancer: Targets and Therapy is an international, peer-reviewed, open access journal focusing on gastro-intestinal cancer research, identification of therapeutic targets and the optima use of preventative and integrated treatment interventions to achieve improved outcomes, enhanced survival and quality of life for the

\section{Dovepress}

cancer patient. The manuscript management system is completely online and includes a very quick and fair peer-review system. Visit http://www.dovepress.com/testimonials.php to read real quotes from published authors.

Submit your manuscript here: http://www.dovepress.com/gastro-intestinal-cancer-targets-and-therapy-journal 